\title{
On a Conjecture of Frankl and Füredi
}

\author{
Ameera Chowdhury * \\ Submitted: Nov 8, 2010; Accepted: Feb 27, 2011; Published: Mar 11, 2011 \\ Mathematics Subject Classification: 05D05
}

\begin{abstract}
Frankl and Füredi conjectured that if $\mathcal{F} \subset 2^{X}$ is a non-trivial $\lambda$-intersecting family of size $m$, then the number of pairs $\{x, y\} \in\left(\begin{array}{c}X \\ 2\end{array}\right)$ that are contained in some $F \in \mathcal{F}$ is at least $\left(\begin{array}{c}m \\ 2\end{array}\right)$ [P. Frankl and Z. Füredi. A Sharpening of Fisher's Inequality. Discrete Math., 90(1):103-107, 1991]. We verify this conjecture in some special cases, focusing especially on the case where $\mathcal{F}$ is additionally required to be $k$-uniform and $\lambda$ is small.
\end{abstract}

\section{Introduction}

Let $X$ be an $n$-element set, and let $2^{X}$ denote the family of all subsets of $X$. For $0 \leq k \leq n$, let $\left(\begin{array}{l}X \\ k\end{array}\right)$ denote the family of all subsets of $X$ of cardinality $k$. For a family $\mathcal{F} \subset 2^{X}$, we define the $i$-shadow of $\mathcal{F}$, denoted $\partial^{i} \mathcal{F}$, to consist of those $i$-subsets of $X$ contained in at least one member of $\mathcal{F}$,

$$
\partial^{i} \mathcal{F}:=\left\{E \in\left(\begin{array}{c}
X \\
i
\end{array}\right): E \subset F \in \mathcal{F}\right\} .
$$

A family $\mathcal{F} \subset 2^{X}$ is called $\lambda$-intersecting if we have $\left|F_{1} \cap F_{2}\right|=\lambda$ for any distinct $F_{1}, F_{2} \in \mathcal{F}$. The well-known Fisher's Inequality states that if $\mathcal{F}$ is a $\lambda$-intersecting family of size $m$, then $\left|\partial^{1} \mathcal{F}\right| \geq m$. Inspired by Fisher's Inequality, Frankl and Füredi [7] conjectured a similar inequality for $\left|\partial^{2} \mathcal{F}\right|$. Conjecture 1.1 easily implies Fisher's Inequality since $\left(\begin{array}{c}\left|\partial^{1} \mathcal{F}\right| \\ 2\end{array}\right) \geq\left|\partial^{2} \mathcal{F}\right| \geq\left(\begin{array}{c}m \\ 2\end{array}\right)$ proves $\left|\partial^{1} \mathcal{F}\right| \geq m$.

Conjecture 1.1 (Frankl-Füredi, 1991) Let $\mathcal{F} \subset 2^{X}$ be a $\lambda$-intersecting family of size $m$. If there does not exist $x \in X$ such that $x \in F$ for all $F \in \mathcal{F}$, then

$$
\left|\partial^{2} \mathcal{F}\right| \geq\left(\begin{array}{c}
m \\
2
\end{array}\right)
$$

*Department of Mathematics, University of California San Diego, La Jolla, CA, 92093, USA. E-mail: anchowdh@math.ucsd.edu 
Frankl and Füredi [7] verified Conjecture 1.1 when $\lambda=1$. While this paper appears to be the first to consider Conjecture 1.1 since [7], several special cases of Conjecture 1.1 had already been proved before [7] was published. For example, Ryser [13], Woodall [16], and Babai [1] showed Conjecture 1.1 is true when $m=n$. Majindar [11] proved Conjecture 1.1 for regular $\lambda$-intersecting families.

Notation: We say $\mathcal{F}$ is $k$-uniform if $\mathcal{F} \subset\left(\begin{array}{l}X \\ k\end{array}\right)$. For $x \in X$, the degree of $x$, denoted $\operatorname{deg}(x)$, is defined to be the number of sets in $\mathcal{F}$ that contain $x$. We can delete $x \in X$ with $\operatorname{deg}(x)=0$, so we always assume $\left|\partial^{1} \mathcal{F}\right|=|X|=n$. We say $\mathcal{F}$ is $r$-regular if $\operatorname{deg}(x)=r$ for all $x \in X$. We say that $\mathcal{F}$ is a sunflower if $\operatorname{deg}(x) \in\{1,|\mathcal{F}|\}$ for all $x \in X$. We say $\mathcal{F}$ is trivial if there exists $x \in X$ with $\operatorname{deg}(x)=|\mathcal{F}|$, and is non-trivial otherwise. For a subset $S \subset X$ we define the co-degree of $S$, denoted codeg $(S)$, to be the number of sets in $\mathcal{F}$ that contain $S$. For a family $\mathcal{F} \subset\left(\begin{array}{l}X \\ k\end{array}\right)$ and $x \in X$, the link of $x$ is the family $L(x):=\{A \subset X:|A|=k-1, A \cup\{x\} \in \mathcal{F}\}$. A $\lambda$-intersecting family $\mathcal{F} \subset\left(\begin{array}{l}X \\ k\end{array}\right)$ of size $m$ is called a symmetric design if $n=\left|\partial^{1} \mathcal{F}\right|=m$.

The non-triviality restriction in Conjecture 1.1 is necessary. For example, if $\mathcal{F} \subset\left(\begin{array}{l}X \\ k\end{array}\right)$ is a $\lambda$-intersecting sunflower of size $m$, then $\left|\partial^{2} \mathcal{F}\right| \leq m\left(\begin{array}{c}k \\ 2\end{array}\right)<\left(\begin{array}{c}m \\ 2\end{array}\right)$ if $m>k(k-1)+1$. We note that Conjecture 1.1 is equivalent to the seemingly stronger statement that if $\mathcal{F} \subset 2^{X}$ is a $\lambda$-intersecting family of size $m$ that is not a sunflower, then $\left|\partial^{2} \mathcal{F}\right| \geq\left(\begin{array}{c}m \\ 2\end{array}\right)$. Fisher's inequality and its variants are usually proved by linear independence arguments [2]; one difficulty in proving Conjecture 1.1 in this way is understanding how to interpret the non-triviality restriction in a linear algebra setting.

The main results of this paper verify Conjecture 1.1 in the following cases. We will use Theorem 1.2 to prove Theorem 1.3.

Theorem 1.2 Let $\mathcal{F} \subset 2^{X}$ be a $\lambda$-intersecting family of size $m$. If $\mathcal{F}$ satisfies

$$
\sum_{F \in \mathcal{F}}\left(\begin{array}{c}
|F| \\
2
\end{array}\right) \geq \sum_{x \in X}\left(\begin{array}{c}
\operatorname{deg}(x) \\
2
\end{array}\right)=\lambda\left(\begin{array}{c}
m \\
2
\end{array}\right),
$$

then $\left|\partial^{2} \mathcal{F}\right| \geq\left(\begin{array}{c}m \\ 2\end{array}\right)$. Moreover, if $\lambda \geq 2$ and $\mathcal{F} \subset\left(\begin{array}{l}X \\ k\end{array}\right)$ is also $k$-uniform, then we have $\left|\partial^{2} \mathcal{F}\right|=\left(\begin{array}{c}m \\ 2\end{array}\right)$ if and only if $\mathcal{F}$ is a symmetric design.

Note that if $\mathcal{F} \subset\left(\begin{array}{l}X \\ k\end{array}\right)$ is a $\lambda$-intersecting family of size $m$, then (1.1) is equivalent to

$$
m \leq \frac{k(k-1)}{\lambda}+1
$$

Theorem 1.3 Let $\mathcal{F} \subset\left(\begin{array}{l}X \\ k\end{array}\right)$ be a non-trivial $\lambda$-intersecting family of size $m$.

(i) If $\lambda=2$, then $\left|\partial^{2} \mathcal{F}\right| \geq\left(\begin{array}{c}m \\ 2\end{array}\right)$ and equality holds if and only if $\mathcal{F}$ is a symmetric design.

(ii) If $\lambda=3$ and $k \notin\{8,11\}$, then $\left|\partial^{2} \mathcal{F}\right| \geq\left(\begin{array}{c}m \\ 2\end{array}\right)$ and equality holds if and only if $\mathcal{F}$ is a symmetric design. 
In light of (1.2), it is interesting to note that Stanton and Mullin [14] once conjectured that if $\mathcal{F} \subset\left(\begin{array}{l}X \\ k\end{array}\right)$ is a non-trivial $\lambda$-intersecting family of size $m$, then (1.2) holds. Had this conjecture been true, Theorem 1.2 would have implied that Conjecture 1.1 is true for uniform families as well as characterized the case of equality. Unfortunately, Hall [10] proved that Stanton and Mullin's conjecture is true only for $\lambda \in\{1,2\}$ and produced counterexamples for every $\lambda \geq 3$.

Since (1.1) and (1.2) are equivalent for uniform families, Hall's proof of Stanton and Mullin's conjecture for $\lambda=2$ shows that (1.1) is true for uniform, non-trivial, 2intersecting families. Combined with Theorem 1.2, Hall's result proves Theorem 1.3 (i). If (1.1) were true for every non-trivial 2-intersecting family, then Theorem 1.2 would imply that Conjecture 1.1 is true for $\lambda=2$. We exhibit one non-trivial 2-intersecting family that does not satisfy (1.1), but feel that this may be the only counterexample. Ryser [13] showed that there is a unique non-uniform 2-intersecting family with size $m=n$ :

$$
\hat{\mathcal{F}}:=\{\{1,2,4\},\{1,4,6,7\},\{1,2,5,7\},\{1,2,3,6\},\{2,3,4,7\},\{1,3,4,5\},\{2,4,5,6\}\} .
$$

It is easily seen that $\sum_{F \in \hat{\mathcal{F}}}\left(\begin{array}{c}|F| \\ 2\end{array}\right)=39$ while $2\left(\begin{array}{c}m \\ 2\end{array}\right)=42$. We conjecture that $\hat{\mathcal{F}}$ is the only non-trivial 2-intersecting family for which (1.1) does not hold.

Conjecture 1.4 If $\mathcal{F} \subset 2^{X}$ is a non-trivial 2-intersecting family of size $m$ and $\mathcal{F} \neq \hat{\mathcal{F}}$, then (1.1) holds.

Frankl and Füredi [7] showed (1.1) holds for all non-trivial 1-intersecting families using an argument similar to that of de Bruijn and Erdös [4]. This summarizes the proof of Conjecture 1.1 when $\lambda=1$ as the proof of Theorem 1.2 is trivial in this case.

Theorem 1.2 implies that a uniform counterexample to Conjecture 1.1 is also a counterexample to Stanton and Mullin's conjecture. It is not difficult to see that Hall's counterexamples to Stanton and Mullin's conjecture do not give counterexamples to Conjecture 1.1; for definitions see [10]. Hence, we can view Conjecture 1.1 as a weakening of Stanton and Mullin's conjecture.

Another weakening of Stanton and Mullin's conjecture is Conjecture 1.5, which is due to Hall [10]. Together with Theorem 1.2, we see that Conjecture 1.5 would imply that Conjecture 1.1 is true if $\mathcal{F}$ is additionally required to be $k$-uniform and $k$ is sufficiently large. Deza [6] showed that $k_{1}=2$; Hall [10] showed that $k_{2}=3$; our proof of Theorem 1.3 shows that $k_{3} \leq 12$.

Conjecture 1.5 (Hall, 1977) For each $\lambda \in \mathbb{Z}^{+}$, there exists a $k_{\lambda} \in \mathbb{Z}^{+}$such that if $k \geq k_{\lambda}$ and $\mathcal{F} \subset\left(\begin{array}{l}X \\ k\end{array}\right)$ is a non-trivial $\lambda$-intersecting family of size $m$, then (1.2) holds.

It is natural to wonder if the obvious analog of Conjecture 1.1 for higher shadows holds. By considering $\lambda$-blowups of projective planes of order $q$ when $q$ is large enough, we have infinitely many nontrivial $\lambda$-intersecting families $\mathcal{F}$ satisfying $\left|\partial^{i} \mathcal{F}\right|<\left(\begin{array}{c}m \\ i\end{array}\right)$ for each $i \geq 3$ and each $\lambda \in \mathbb{Z}^{+}$. 


\section{Proof of Theorem 1.2}

We use linear programming duality to prove Theorem 1.2. We will use Theorem 1.2 to prove Theorem 1.3 in Section 3.

Proof of Theorem 1.2. When $\lambda=1$, the proof of Theorem 1.2 is trivial because $\left|\partial^{2} \mathcal{F}\right|$ equals the left hand side of (1.1). We therefore assume that $\lambda \geq 2$. Let $\mathcal{F} \subset 2^{X}$ be a $\lambda$-intersecting family of size $m$. Let $a_{i}$ denote the number of pairs $\{x, y\} \in\left(\begin{array}{c}X \\ 2\end{array}\right)$ with $\operatorname{codeg}(\{x, y\})=i$, and observe that the following identities hold

$$
\sum_{i \geq 1} i a_{i}=\sum_{F \in \mathcal{F}}\left(\begin{array}{c}
|F| \\
2
\end{array}\right), \quad \sum_{i \geq 1}\left(\begin{array}{l}
i \\
2
\end{array}\right) a_{i}=\left(\begin{array}{l}
\lambda \\
2
\end{array}\right)\left(\begin{array}{c}
m \\
2
\end{array}\right) .
$$

The first follows from counting pairs $(\{x, y\}, F)$ where $\{x, y\} \in\left(\begin{array}{c}X \\ 2\end{array}\right), F \in \mathcal{F}$, and $\{x, y\} \subset F$. The second follows from counting pairs $\left(\{x, y\},\left\{F_{1}, F_{2}\right\}\right)$ where $\{x, y\} \in\left(\begin{array}{c}X \\ 2\end{array}\right)$, $\left\{F_{1}, F_{2}\right\} \subset \mathcal{F}$, and $\{x, y\} \subset F_{1} \cap F_{2}$. Consequently, $\left(a_{1}, \ldots, a_{m}\right)$ is a feasible solution to the following linear program with objective value $\left|\partial^{2} \mathcal{F}\right|$ :

$$
\begin{aligned}
& \text { Minimize } \\
& \sum_{i=1}^{m} z_{i} \\
& \text { subject to: } \\
& \sum_{i \geq 1} i z_{i}=\sum_{F \in \mathcal{F}}\left(\begin{array}{c}
|F| \\
2
\end{array}\right) \\
& \sum_{i \geq 1}\left(\begin{array}{l}
i \\
2
\end{array}\right) z_{i}=\left(\begin{array}{l}
\lambda \\
2
\end{array}\right)\left(\begin{array}{c}
m \\
2
\end{array}\right) \\
& z_{i} \geq 0, \quad i \in\{1, \ldots, m\} \text {. }
\end{aligned}
$$

The dual of this linear program is:

$$
\begin{array}{ll}
\text { Maximize } & \left(\begin{array}{l}
\lambda \\
2
\end{array}\right)\left(\begin{array}{c}
m \\
2
\end{array}\right) x+\left(\sum_{F \in \mathcal{F}}\left(\begin{array}{c}
|F| \\
2
\end{array}\right)\right) y \\
\text { subject to: } & \left(\begin{array}{c}
i \\
2
\end{array}\right) x+i y \leq 1, \quad i \in\{1, \ldots, m\} .
\end{array}
$$

The feasible region of the dual linear program (2.4) has extreme points given by

$$
\left(-\frac{1}{\left(\begin{array}{c}
j+1 \\
2
\end{array}\right)}, \frac{2}{j+1}\right), j \in\{1, \ldots, m-1\} .
$$

If $\mathcal{F}$ satisfies (1.1), then setting $j=\lambda-1$ in (2.5) yields

$$
\left|\partial^{2} \mathcal{F}\right| \geq\left(\begin{array}{c}
\lambda \\
2
\end{array}\right)\left(\begin{array}{c}
m \\
2
\end{array}\right)\left(-\frac{1}{\left(\begin{array}{c}
\lambda \\
2
\end{array}\right)}\right)+\left(\sum_{F \in \mathcal{F}}\left(\begin{array}{c}
|F| \\
2
\end{array}\right)\right)\left(\begin{array}{c}
2 \\
\lambda
\end{array}\right) \geq\left(\begin{array}{c}
m \\
2
\end{array}\right),
$$


as desired. Finally, note that the equality in (1.1) follows from counting pairs $\left(x,\left\{F_{1}, F_{2}\right\}\right)$ such that $\left\{F_{1}, F_{2}\right\} \subset \mathcal{F}$ and $x \in F_{1} \cap F_{2}$.

We now assume $\lambda \geq 2$ and $\mathcal{F} \subset\left(\begin{array}{c}X \\ k\end{array}\right)$ is also $k$-uniform, and prove that $\left|\partial^{2} \mathcal{F}\right|=\left(\begin{array}{c}m \\ 2\end{array}\right)$ if and only if $\mathcal{F}$ is a symmetric design. Ryser [13], Woodall [16], and Babai [1] showed that if $\mathcal{F} \subset 2^{X}$ is a $\lambda$-intersecting family of size $m=\left|\partial^{1} \mathcal{F}\right|=n$, then $\left|\partial^{2} \mathcal{F}\right|=\left(\begin{array}{c}m \\ 2\end{array}\right)$. Conversely, suppose $\left|\partial^{2} \mathcal{F}\right|=\left(\begin{array}{c}m \\ 2\end{array}\right)$ and let $a_{i}$ denote the number of pairs $\{x, y\} \in\left(\begin{array}{c}X \\ 2\end{array}\right)$ with codeg $(\{x, y\})=i$. We will show that $\mathcal{F}$ is $k$-regular, which immediately implies that $\mathcal{F}$ is a symmetric design. By $(2.6)$, we see that equality holds in $(1.2),\left(a_{1}, \ldots, a_{m}\right)$ is an optimal solution to the primal linear program (2.3), and (2.5) with $j=\lambda-1$ is an optimal solution to the dual linear program (2.4). By complementary slackness, this implies $a_{i}=0$ or $\left(\begin{array}{l}i \\ 2\end{array}\right)\left(-1 /\left(\begin{array}{l}\lambda \\ 2\end{array}\right)\right)+i(2 / \lambda)=1$. Hence $a_{i}=0$ for $i \notin\{\lambda-1, \lambda\}$ for $i \in\{1, \ldots m\}$. The constraints in the primal linear program (2.3) imply $a_{\lambda-1}+a_{\lambda}=\left(\begin{array}{c}m \\ 2\end{array}\right)$ and $\left(\begin{array}{c}\lambda-1 \\ 2\end{array}\right) a_{\lambda-1}+\left(\begin{array}{c}\lambda \\ 2\end{array}\right) a_{\lambda}=\left(\begin{array}{c}\lambda \\ 2\end{array}\right)\left(\begin{array}{c}m \\ 2\end{array}\right)$, so $a_{\lambda-1}=0$ and $a_{\lambda}=\left(\begin{array}{c}m \\ 2\end{array}\right)$.

Let $x \in X$ and count pairs $(y, F)$ such that $\{x, y\} \subset F$. Since $a_{\lambda}=\left(\begin{array}{c}m \\ 2\end{array}\right)$, we have

$$
\lambda\left|\partial^{1} L(x)\right|=(k-1) \operatorname{deg}(x) \text { so }\left|\partial^{1} L(x)\right|=\frac{k-1}{\lambda} \operatorname{deg}(x) .
$$

We will give a lower bound on $\left|\partial^{1} L(x)\right|$ in terms of $\operatorname{deg}(x)$ that will allow us to prove that $\mathcal{F}$ is $k$-regular. For $x \in X$, let $b_{x, i}$ denote the number of vertices $y \in X$ such that $\operatorname{codeg}(\{x, y\})=i$ and observe that the following identities hold

$$
\sum_{i \geq 1} i b_{x, i}=(k-1) \operatorname{deg}(x), \quad \sum_{i \geq 1}\left(\begin{array}{l}
i \\
2
\end{array}\right) b_{x, i}=(\lambda-1)\left(\begin{array}{c}
\operatorname{deg}(x) \\
2
\end{array}\right) .
$$

The first follows from counting pairs $(y, F)$ where $\{x, y\} \in\left(\begin{array}{c}X \\ 2\end{array}\right), F \in \mathcal{F}$, and $\{x, y\} \subset F$. The second follows from counting pairs $\left(y,\left\{F_{1}, F_{2}\right\}\right)$ where $\{x, y\} \in\left(\begin{array}{c}X \\ 2\end{array}\right),\left\{F_{1}, F_{2}\right\} \subset \mathcal{F}$, and $\{x, y\} \subset F_{1} \cap F_{2}$. Consequently, $\left(b_{x, 1}, \ldots, b_{x, m}\right)$ is a feasible solution to the following linear program with objective value $\left|\partial^{1} L(x)\right|$ :

$$
\begin{aligned}
& \text { Minimize } \\
& \qquad \begin{array}{l}
\sum_{i=1}^{m} i w_{i}=(k-1) \operatorname{deg}(x) \\
\text { subject to: } w_{i}
\end{array} \\
& \qquad \sum_{i \geq 1}\left(\begin{array}{l}
i \\
2
\end{array}\right) w_{i}=(\lambda-1)\left(\begin{array}{c}
\operatorname{deg}(x) \\
2
\end{array}\right) \\
& w_{i} \geq 0, \quad i \in\{1, \ldots, m\} .
\end{aligned}
$$

The dual of this linear program is:

$$
\begin{array}{lr}
\text { Maximize } & (\lambda-1)\left(\begin{array}{c}
\operatorname{deg}(x) \\
2
\end{array}\right) y+(k-1) \operatorname{deg}(x) z \\
\text { subject to: } & \left(\begin{array}{c}
i \\
2
\end{array}\right) y+i z \leq 1, \quad i \in\{1, \ldots, m\} .
\end{array}
$$


Since (2.5) with $j=\lambda-1$ is a feasible solution, using (2.7) yields

$$
\frac{k-1}{\lambda} \operatorname{deg}(x)=\left|\partial^{1} L(x)\right| \geq \operatorname{deg}(x)\left(\frac{2(k-1)}{\lambda}-(\lambda-1) \frac{\operatorname{deg}(x)-1}{2}\left(\begin{array}{l}
\lambda \\
2
\end{array}\right)^{-1}\right) .
$$

Hence, $\operatorname{deg}(x) \geq k$ for each $x \in X$. On the other hand, let $F \in \mathcal{F}$ and count pairs $\left(x, F^{\prime}\right)$ such that $F \neq F^{\prime} \in \mathcal{F}$ and $x \in F \cap F^{\prime}$. Since equality holds in (1.2), we have

$$
k^{2} \leq \sum_{x \in F} \operatorname{deg}(x)=\lambda(m-1)+k=k^{2}
$$

so $\operatorname{deg}(x)=k$ for all $x \in X$. Hence $\mathcal{F}$ is $k$-regular and is thus a symmetric design.

\section{Proof of Theorem 1.3}

In light of (1.1) and (1.2), we are interested in upper bounds on the sizes of non-trivial $\lambda$-intersecting families $\mathcal{F}$ that depend only on the sizes of the sets in $\mathcal{F}$. One of the first results of this kind is Deza's theorem [6], which bounds the size of $\lambda$-intersecting families that are not sunflowers. In the case when $\mathcal{F} \subset\left(\begin{array}{l}X \\ k\end{array}\right)$ is $k$-uniform, the upper bound on $m$ in (3.8) is bigger than the upper bound on $m$ in (1.2) by a factor of roughly $\lambda$.

Theorem 3.1 (Deza, 1974) Let $\mathcal{F} \subset 2^{X}$ be a $\lambda$-intersecting family of size $m$ that is not a sunflower. Define $K:=\max _{F \in \mathcal{F}}|F|$. Then

$$
m \leq \max \{\lambda(\lambda+1)+1,(K-\lambda)((K-\lambda)+1)+1\} .
$$

Since non-triviality is a stronger restriction on $\mathcal{F}$ than not being a sunflower, it is plausible that (3.8) could be improved for non-trivial $\mathcal{F}$. Frankl and Füredi [7] did exactly this when they showed that (1.1) holds for all non-trivial 1-intersecting families. We mentioned in the introduction that Stanton and Mullin [14] conjectured that (3.8) could be improved to (1.2) if $\mathcal{F}$ is non-trivial and $k$-uniform; Theorem 3.1 verifies Stanton and Mullin's conjecture for $\lambda=1$ and Hall proved Stanton and Mullin's conjecture when $\lambda=2$.

Theorem 3.2 (Hall, 1977) If $\mathcal{F} \subset\left(\begin{array}{l}X \\ k\end{array}\right)$ is a non-trivial 2-intersecting family of size $m$, then

$$
m \leq\left(\begin{array}{l}
k \\
2
\end{array}\right)+1
$$

We adapt Hall's proof of Theorem 3.2 to prove Theorem 1.3. (For the reader's convenience, we first reproduce Hall's proof of Theorem 3.2.) In our proof of Theorem 1.3, we will use the fact that if $\mathcal{F} \subset\left(\begin{array}{l}X \\ k\end{array}\right)$ is a $\lambda$-intersecting family, then $\operatorname{deg}(x)$ does not lie in a certain interval. Deza [5] showed that if $\mathcal{F} \subset\left(\begin{array}{l}X \\ k\end{array}\right)$ is a $\lambda$-intersecting family of size $m$ then, for all $x \in X$,

$$
\operatorname{deg}(x)(m+1-\operatorname{deg}(x)) \leq \max \{\lambda, k-\lambda\}(m+1) .
$$


McCarthy and Vanstone [12] adapted an argument of Connor [3], and improved this bound; they gave the following restriction on $\operatorname{deg}(x)$.

Theorem 3.3 (McCarthy-Vanstone, 1979) Let $\mathcal{F} \subset\left(\begin{array}{l}X \\ k\end{array}\right)$ be a $\lambda$-intersecting family of size $m$.

(i) If $x \in X$ then,

$$
\operatorname{deg}(x)((k-\lambda)+\lambda(m-\operatorname{deg}(x))) \leq(k-\lambda)((k-\lambda)+\lambda m) .
$$

(ii) Let $\{x, y\} \subset\left(\begin{array}{c}X \\ 2\end{array}\right)$ and define

(a) $a_{11}=(k-\lambda)((k-\lambda)+\lambda m)-\operatorname{deg}(x)((k-\lambda)+\lambda(m-\operatorname{deg}(x)))$,

(b) $a_{12}=a_{21}=\lambda \operatorname{deg}(x) \operatorname{deg}(y)-((k-\lambda)+\lambda m) \operatorname{codeg}(\{x, y\})$,

(c) $a_{22}=(k-\lambda)((k-\lambda)+\lambda m)-\operatorname{deg}(y)((k-\lambda)+\lambda(m-\operatorname{deg}(y)))$.

The following determinant is non-negative:

$$
\operatorname{det}\left(\begin{array}{ll}
a_{11} & a_{12} \\
a_{21} & a_{22}
\end{array}\right) \geq 0 .
$$

We now reproduce Hall's proof of Theorem 3.2. Note that Hall had originally used (3.9) in his proof, but we will use (3.10) instead since it makes the argument cleaner.

Hall's Proof of Theorem 3.2 Suppose, for a contradiction, that there exists a nontrivial 2-intersecting $\mathcal{F} \subset\left(\begin{array}{l}X \\ k\end{array}\right)$ of size $m>\left(\begin{array}{l}k \\ 2\end{array}\right)+1$. Write

$$
m=\left(\begin{array}{l}
k \\
2
\end{array}\right)+1+\epsilon, \quad \epsilon \in \mathbb{Z}^{+} .
$$

Observe that the left hand side of (3.10) is quadratic in $\operatorname{deg}(x)$ with $\operatorname{roots} \operatorname{deg}(x)=0$ and $\operatorname{deg}(x)=m-1+k / 2$. If there exists an $x \in X$ with $k \leq \operatorname{deg}(x) \leq(m-1+k / 2)-k$, then (3.10) is true for $\operatorname{deg}(x)=k$; together with (3.12), this implies that $\epsilon \leq 0$, which is impossible. Hence, for all $x \in X$, either

$$
\operatorname{deg}(x) \leq k-1 \text { or } \operatorname{deg}(x) \geq m-\left\lceil\frac{k}{2}\right\rceil
$$

We say a vertex $x \in X$ with $\operatorname{deg}(x) \leq k-1$ is light and is heavy if $\operatorname{deg}(x) \geq m-\lceil k / 2\rceil$.

By (3.12), for any $F \in \mathcal{F}$, we have

$$
\sum_{x \in F} \operatorname{deg}(x)=2(m-1)+k=k^{2}+2 \epsilon .
$$


Since the average degree of a vertex in $F \in \mathcal{F}$ is greater than $k$, every set $F \in \mathcal{F}$ contains a heavy vertex. As $\mathcal{F}$ is non-trivial, there are at least two heavy vertices $x_{1}, x_{2}$. Define

$$
\begin{aligned}
s:=\left|\left\{F \in \mathcal{F}:\left\{x_{1}, x_{2}\right\} \subset \mathcal{F}\right\}\right|, & t:=\left|\left\{F \in \mathcal{F}: x_{1} \in F, x_{2} \notin F\right\}\right|, \\
u:=\left|\left\{F \in \mathcal{F}: x_{1} \notin F, x_{2} \in F\right\}\right|, & v:=\left|\left\{F \in \mathcal{F}: x_{1}, x_{2} \notin F\right\}\right| .
\end{aligned}
$$

We have $s \leq k-1$ because $\lambda=2$ and $\mathcal{F}$ is non-trivial. Since $u+v$ and $t+v$ count the number of sets $F \in \mathcal{F}$ not on $x_{1}, x_{2}$ respectively, (3.13) yields $t+v, u+v \leq\lceil k / 2\rceil$. Consequently (3.12) implies,

$$
\left(\begin{array}{l}
k \\
2
\end{array}\right)+1+\epsilon=m=s+t+u+v \leq s+(t+v)+(u+v) \leq(k-1)+2\left\lceil\frac{k}{2}\right\rceil \leq 2 k .
$$

As $\epsilon \in \mathbb{Z}^{+}$, we have a contradiction for $k \geq 5$. For $k=4$, Theorem 3.1 yields $m \leq 7$, so we have a contradiction in this case too. We have shown that if $\mathcal{F} \subset\left(\begin{array}{l}X \\ k\end{array}\right)$ is a non-trivial 2-intersecting family of size $m$ then $m \leq\left(\begin{array}{l}k \\ 2\end{array}\right)+1$.

For larger $\lambda$, if we knew that a non-trivial $\lambda$-intersecting $\mathcal{F} \subset\left(\begin{array}{l}X \\ k\end{array}\right)$ that does not satisfy (1.2) has at least $\lambda$ heavy vertices, then Hall's argument would yield a proof of Conjecture 1.5. Unfortunately, Hall's averaging argument only shows that any non-trivial $\lambda$-intersecting $\mathcal{F} \subset\left(\begin{array}{l}X \\ k\end{array}\right)$ that does not satisfy (1.2) has at least two heavy vertices. In the proof of Theorem 1.3, we expend a lot of effort to eliminate the possibility that there are exactly two heavy vertices when $\lambda=3$; the key difficulty is getting a good bound on the number of sets $F \in \mathcal{F}$ that contain both the heavy vertices.

Proof of Theorem 1.3. We observe that Theorem 3.2 together with Theorem 1.2 yields Theorem 1.3 (i).

For the rest of the proof, we assume that $\lambda=3$. We will show that if $\mathcal{F} \subset\left(\begin{array}{l}X \\ k\end{array}\right)$ is a non-trivial 3-intersecting family, where $k \notin\{8,11\}$, then (1.2) holds. Theorem 1.2 then implies that $\left|\partial^{2} \mathcal{F}\right| \geq\left(\begin{array}{c}m \\ 2\end{array}\right)$ and that equality holds if and only if $\mathcal{F}$ is a symmetric design. First suppose $k<6$. It is not difficult to see that if $\mathcal{F}$ is a non-trivial $k$-uniform 3 -intersecting family of size $m$, where $k \in\{4,5\}$, then $m \leq 5$; for proofs of these results in a more general setting see [8], [9], and [15]. Hence, (1.2) holds when $k<6$.

Suppose, for a contradiction, that $k \geq 12$ and $\mathcal{F} \subset\left(\begin{array}{l}X \\ k\end{array}\right)$ is a non-trivial 3-intersecting family of size $m$ for which (1.2) does not hold. Write

$$
m=\frac{k(k-1)}{3}+1+\epsilon, \quad \epsilon>0 .
$$

Note that the left hand side of (3.10) is quadratic in $\operatorname{deg}(x)$ with $\operatorname{roots} \operatorname{deg}(x)=0$ and $\operatorname{deg}(x)=m-1+k / 3$. If there exists an $x \in X$ with $k \leq \operatorname{deg}(x) \leq(m-1+k / 3)-k$, then (3.10) is true for $\operatorname{deg}(x)=k$; together with (3.15), this implies that $\epsilon \leq 0$, which is impossible. Hence, for all $x \in X$, either

$$
\operatorname{deg}(x) \leq k-1 \text { or } \operatorname{deg}(x) \geq m-\left\lceil\frac{2 k}{3}\right\rceil \geq m-\frac{2 k+2}{3} .
$$


Following Hall [10], we say a vertex $x \in X$ is $\operatorname{light}$ if $\operatorname{deg}(x) \leq k-1$ and is heavy if $\operatorname{deg}(x) \geq m-\lceil 2 k / 3\rceil$.

By (3.15), for any $F \in \mathcal{F}$, we have

$$
\sum_{x \in F} \operatorname{deg}(x)=3(m-1)+k=k^{2}+3 \epsilon .
$$

Since the average degree of a vertex in $F \in \mathcal{F}$ is greater than $k$, every set $F \in \mathcal{F}$ contains a heavy vertex. As $\mathcal{F}$ is non-trivial, there are at least two heavy vertices. We consider two cases, according to whether there are exactly two or greater than two heavy vertices.

Case 1: There are exactly two heavy vertices. Let $x_{1}, x_{2}$ be the heavy vertices. Since $\mathcal{F}$ is non-trivial, there exists a set $F_{1} \in \mathcal{F}$ which contains $x_{1}$ but not $x_{2}$, and there exists a set $F_{2} \in \mathcal{F}$ which contains $x_{2}$ but not $x_{1}$. Let $F_{1} \cap F_{2}:=\left\{y_{1}, y_{2}, y_{3}\right\}$. Define

$$
\begin{aligned}
& s:=\left|\left\{F \in \mathcal{F}:\left\{x_{1}, x_{2}\right\} \subset \mathcal{F}\right\}\right|, \quad t:=\left|\left\{F \in \mathcal{F}: x_{1} \in F, x_{2} \notin F\right\}\right|, \\
& u:=\left|\left\{F \in \mathcal{F}: x_{1} \notin F, x_{2} \in F\right\}\right|,
\end{aligned}
$$

and observe that $m=s+t+u$ since every $F \in \mathcal{F}$ contains a heavy vertex. By (3.16), we have $t, u \leq\lceil 2 k / 3\rceil \leq(2 k+2) / 3$.

We now show how to obtain an upper bound on $s$ in terms of $k$. Observe that any $F \in \mathcal{F}$ that contains $\left\{x_{1}, x_{2}\right\}$ intersects $F_{1} \backslash\left\{x_{1}\right\}$ in a subset of size two. Consequently,

$$
2 s=\sum_{\left\{x_{1}, x_{2}\right\} \subset F}\left|F \cap F_{1} \backslash\left\{x_{1}\right\}\right|=\sum_{w \in F_{1} \backslash\left\{x_{1}\right\}} \operatorname{codeg}\left(x_{1}, x_{2}, w\right) .
$$

We claim that if $w \in X \backslash\left\{x_{1}, x_{2}\right\}$ and there exists an $F \in \mathcal{F}$ such that

$$
\left\{x_{1}, x_{2}\right\} \not \subset F, w \notin F,
$$

then codeg $\left(\left\{x_{1}, x_{2}, w\right\}\right) \leq(k-1) / 2$. Suppose $F^{\prime}, \hat{F} \in \mathcal{F}$ are distinct sets in $\mathcal{F}$ that both contain $\left\{x_{1}, x_{2}, w\right\}$. Since $\lambda=3$, we see that the intersections of $F^{\prime}$ and $\hat{F}$ with $F \backslash\left\{x_{1}, x_{2}\right\}$ must be disjoint subsets of size two. Consequently, $\operatorname{codeg}\left(\left\{x_{1}, x_{2}, w\right\}\right) \leq(k-1) / 2$. Observe that if $w \in F_{1} \backslash\left\{x_{1}, y_{1}, y_{2}, y_{3}\right\}$ then $F_{2}$ is a set in $\mathcal{F}$ that satisfies (3.19). We will consider two subcases according to whether for each $y_{i} \in F_{1} \cap F_{2}$, there exists an $F \in \mathcal{F}$ that satisfies (3.19) for $w=y_{i}$.

Subcase 1: For each $y_{i} \in F_{1} \cap F_{2}$, there exists an $F \in \mathcal{F}$ that satisfies (3.19) for $w=y_{i}$.

Applying (3.16) and (3.18) yields

$$
\frac{k(k-1)}{3}+1+\epsilon=m=s+t+u \leq \frac{(k-1)^{2}}{4}+2\left\lceil\frac{2 k}{3}\right\rceil \leq \frac{3 k^{2}+10 k+19}{12} .
$$

This implies that $k^{2}-14 k-7+12 \epsilon \leq 0$, which is a contradiction for $k \geq 15$ since $\epsilon \geq 1 / 3$. For the remaining values of $k$, we refer the reader to the appendix. 
Subcase 2: There exists a $y_{i} \in F_{1} \cap F_{2}$ for which no $F \in \mathcal{F}$ satisfies (3.19) for $w=y_{i}$.

Observe that if $y_{i}$ is in every $F \in \mathcal{F}$ that does not contain $\left\{x_{1}, x_{2}\right\}$ then, by (3.16), codeg $\left(\left\{x_{1}, x_{2}, y_{i}\right\}\right) \leq k-1-(t+u)$. Suppose that every $F \in \mathcal{F}$, not containing $\left\{x_{1}, x_{2}\right\}$, contains $j$ of the elements $\left\{y_{1}, y_{2}, y_{3}\right\}$ where $j \in\{1,2,3\}$. Applying (3.18) yields

$$
\begin{aligned}
\frac{k(k-1)}{3}+1+\epsilon & =m=s+t+u \\
& \leq \frac{1}{2}\left(\sum_{w \in F_{1} \backslash\left\{x_{1}\right\}} \operatorname{codeg}\left(x_{1}, x_{2}, w\right)\right)+t+u \\
& \leq \frac{1}{2}\left(j(k-1-(t+u))+\frac{(k-1-j)(k-1)}{2}\right)+t+u \\
& =\frac{j}{2}(k-1)+\frac{(k-1-j)(k-1)}{4}+(2-j) \frac{t+u}{2} \\
& \leq \frac{j}{2}(k-1)+\frac{(k-1-j)(k-1)}{4}+(2-j) \frac{2 k+2}{3} \\
& =-\left(\frac{k}{6}+\frac{7}{6}\right) j+\frac{(k-1-j)(k-1)}{4}+\frac{4 k+4}{3} \\
& \leq \frac{3 k^{2}+5 k+8}{12},
\end{aligned}
$$

since the penultimate expression in $(3.21)$ is maximized when $j=1$. This implies that $(k-1)(k-8)+12(\epsilon-1 / 3) \leq 0$, which is a contradiction for $k \geq 9$.

If $k=8$ then $\epsilon=1 / 3$. Observe that $\operatorname{codeg}\left(x_{1}, x_{2}, w\right) \leq 3$ if $w$ is not one of the $j$ special vertices in $\left\{y_{1}, y_{2}, y_{3}\right\}$; in the bound for $s$ in (3.21), we use the weaker bound codeg $\left(x_{1}, x_{2}, w\right) \leq 7 / 2$ for vertices $w$ that are not one of the $j$ special vertices in $\left\{y_{1}, y_{2}, y_{3}\right\}$. If we replace the weaker bound on codeg $\left(x_{1}, x_{2}, w\right)$ by the tighter bound, then we get a contradiction for $k=8$ as well. Finally, if $k \in\{6,7\}$, then $\epsilon \in \mathbb{Z}^{+}$so we also get a contradiction in this case.

Case 2: There are greater than two heavy vertices. Let $x_{1}, x_{2}, x_{3}$ be three heavy vertices. Define

$$
\begin{array}{rrrl}
s:=\left|\left\{F \in \mathcal{F}:\left\{x_{1}, x_{2}, x_{3}\right\} \subset \mathcal{F}\right\}\right|, & t:=\left|\left\{F \in \mathcal{F}: x_{1} \in F, x_{2}, x_{3} \notin F\right\}\right|, \\
u:=\left|\left\{F \in \mathcal{F}: x_{2} \in F, x_{1}, x_{3} \notin F\right\}\right|, & v:=\left|\left\{F \in \mathcal{F}: x_{3} \in F, x_{1}, x_{2} \notin F\right\}\right|, \\
w:=\left|\left\{F \in \mathcal{F}: x_{1}, x_{2} \in F, x_{3} \notin F\right\}\right|, & x:=\left|\left\{F \in \mathcal{F}: x_{1}, x_{3} \in F, x_{2} \notin F\right\}\right|, \\
y:=\left|\left\{F \in \mathcal{F}: x_{2}, x_{3} \in F, x_{1} \notin F\right\}\right|, & z:=\left|\left\{F \in \mathcal{F}: x_{1}, x_{2}, x_{3} \notin F\right\}\right| .
\end{array}
$$

By counting the number of sets not containing $x_{1}, x_{2}$, or $x_{3}$ respectively we have

$$
u+v+y+z, t+v+x+z, t+u+w+z \leq\left\lceil\frac{2 k}{3}\right\rceil \leq \frac{2 k+2}{3},
$$


by (3.16). As $\lambda=3$ and $\mathcal{F}$ is non-trivial, we have $s \leq k-2$. Therefore (3.22) implies,

$$
\begin{aligned}
\frac{k(k-1)}{3}+1+\epsilon & =m=s+t+u+v+w+x+y+z \\
& \leq s+(u+v+y+z)+(t+v+x+z)+(t+u+w+z) \\
& \leq(k-2)+3\left\lceil\frac{2 k}{3}\right\rceil \leq(k-2)+(2 k+2)=3 k .
\end{aligned}
$$

This implies $k^{2}-10 k+3+3 \epsilon \leq 0$, so we have a contradiction for $k \geq 10$ since $\epsilon \geq 1 / 3$. For the remaining values of $k$, we refer the reader to the appendix.

We have shown that if $\mathcal{F} \subset\left(\begin{array}{l}X \\ k\end{array}\right)$ is a non-trivial 3-intersecting family of size $m$ and $k \notin\{8,11\}$, then $\mathcal{F}$ satisfies (1.2). By Theorem 1.2, this implies that if $\mathcal{F}$ satisfies the hypotheses of Theorem 1.3 (ii), then $\left|\partial^{2} \mathcal{F}\right| \geq\left(\begin{array}{c}m \\ 2\end{array}\right)$ and equality holds if and only if $\mathcal{F}$ is a symmetric design.

\section{Appendix}

Here, we collect some computations that are needed to verify Theorem 1.3 for small values of $k$. We regret that we could not prove Theorem 1.3 for all values of $k$. The missing cases are $k=11, m=40$ in Case 1 , Subcase 1 and $k=8, m=20$ in Case 2.

Case Analysis for Case 1, Subcase 1:

If $k=14$, then codeg $\left(x_{1}, x_{2}, w\right) \leq 6$ for $w \in F_{1} \backslash\left\{x_{1}\right\}$ so (3.18) yields $s \leq 39$. Using this value for $s$ in (3.20) yields $\epsilon<0$, which contradicts (3.15).

If $k=13$, then the penultimate inequality in (3.20) yields that $m=54, s=36$, and $t=u=9$. Using these values in (3.11) yields a contradiction.

If $k=12$, then codeg $\left(x_{1}, x_{2}, w\right) \leq 5$ for $w \in F_{1} \backslash\left\{x_{1}\right\}$ so (3.18) yields $s \leq 27$. Using this value for $s$ in (3.20) yields $\epsilon<0$, which contradicts (3.15).

If $k=11$, then $(3.20)$ yields $m \in\{38,39,40,41\}$. If we add the constraint $z_{s}=1$ to (2.3), then the dual linear program becomes

$$
\begin{array}{r}
\quad 3\left(\begin{array}{c}
m \\
2
\end{array}\right) w+\left(\begin{array}{c}
k \\
2
\end{array}\right) m x+y \\
\text { Maximize } \\
\text { subject to: } \quad\left(\begin{array}{l}
i \\
2
\end{array}\right) w+i x \leq 1, \quad i \in\{1, \ldots, m\} \backslash\{s\} \\
\left(\begin{array}{l}
s \\
2
\end{array}\right) w+s x+y \leq 1 .
\end{array}
$$

and any feasible solution to (4.24) is a lower bound on $\left|\partial^{2} \mathcal{F}\right|$. If $m=38$, then (3.20) implies $s \geq 22$ because $t, u \leq 8$. Observe that $\left(\begin{array}{l}p \\ 2\end{array}\right)\left(-\frac{1}{3}\right)+p\left(\frac{2}{3}\right) \leq\left(\begin{array}{l}q \\ 2\end{array}\right)\left(-\frac{1}{3}\right)+q\left(\frac{2}{3}\right)$ if $p>q \geq 2$. We have that $\left(\begin{array}{c}22 \\ 2\end{array}\right)\left(-\frac{1}{3}\right)+22\left(\frac{2}{3}\right)+63 \frac{1}{3}=1$, so the previous inequality implies 
that $\left(-1 / 3,2 / 3,63 \frac{1}{3}\right)$ is always a feasible solution to (4.24) for $m=38$ and $k=11$. Hence, $\left|\partial^{2} \mathcal{F}\right|>\left(\begin{array}{c}m \\ 2\end{array}\right)$, which contradicts our initial assumption. A similar argument eliminates the case $m=39$. If $m=41$, then $s=25$ and $t=u=8$ so $\operatorname{deg}\left(x_{1}\right)=33$; this contradicts Theorem 3.3.

If $k=10$, then the penultimate inequality in (3.20) yields that $m=32$ and $s=18$. Since $\left(\begin{array}{c}18 \\ 2\end{array}\right)\left(-\frac{1}{3}\right)+18\left(\frac{2}{3}\right)+40=1$, we have $(-1 / 3,2 / 3,40)$ is a feasible solution to $(4.24)$ for $m=32$ and $k=10$. Consequently, $\left|\partial^{2} \mathcal{F}\right|>\left(\begin{array}{c}m \\ 2\end{array}\right)$, which contradicts our initial assumption.

If $k=9$, then (3.20) yields $\epsilon \in\{1,2,3\}$ and $s \leq 16$. We consider the cases $\epsilon=1$ and $\epsilon \in\{2,3\}$ separately.

$\underline{\epsilon=1}$ : By (3.16), we have $s \geq 14$ and $t, u \leq 6$. Note that $\left(\begin{array}{c}15 \\ 2\end{array}\right)\left(-\frac{1}{3}\right)+15\left(\frac{2}{3}\right)+26=1$ so $\left.\overline{\left(-\frac{1}{3}, \frac{2}{3}\right.}, 26\right)$ is a feasible solution to (4.24) for $m=26, k=9$, and $s \geq 15$. If $s=14$, then $t=u=6$ so $\operatorname{deg}\left(x_{1}\right)=\operatorname{deg}\left(x_{2}\right)=20$. Observe that (3.16) and (3.17) imply

$$
84=\sum_{x \in F_{1}} \operatorname{deg}(x)=\operatorname{deg}\left(x_{1}\right)+\sum_{x_{1} \neq x \in F_{1}} \operatorname{deg}(x) \leq 20+8(8)=84
$$

hence, if $w$ is a light vertex in a set in $\mathcal{F}$ that doesn't contain both $x_{1}, x_{2}$, then $\operatorname{deg}(w)=8$. Now suppose that $z$ is a light vertex that is only contained in sets that contain both $x_{1}, x_{2}$; that is $\operatorname{deg}(z)=\operatorname{codeg}\left(x_{1}, x_{2}, z\right)$. Since $F_{1}$ satisfies (3.19) for $w=z$, we see $\operatorname{deg}(z)=\operatorname{codeg}\left(x_{1}, x_{2}, z\right) \leq 4$. Now let $F^{\prime} \in \mathcal{F}$ be a set that contains both $x_{1}, x_{2}$ and observe that

$$
84=\sum_{x \in F^{\prime}} \operatorname{deg}(x)=\operatorname{deg}\left(x_{1}\right)+\operatorname{deg}\left(x_{2}\right)+\sum_{z \in F^{\prime} \backslash\left\{x_{1}, x_{2}\right\}} \operatorname{deg}(z)=20+20+\sum_{z \in F^{\prime} \backslash\left\{x_{1}, x_{2}\right\}} \operatorname{deg}(z) .
$$

For $z \in F^{\prime} \backslash\left\{x_{1}, x_{2}\right\}$, we have $\operatorname{deg}(z) \leq 4$ or $\operatorname{deg}(z)=8$ so either $F^{\prime}$ contains four vertices of degree eight and three vertices of degree four or five vertices of degree eight and two vertices whose degrees sum to four. Hence for $x \in X$, we have $\operatorname{deg}(x) \in\{1,2,3,4,8,20\}$. Let $n_{i}$ denote the number of vertices of degree $i$. Also define

$$
\begin{aligned}
& m_{1}:=\mid\left\{F \in \mathcal{F}:\left\{x_{1}, x_{2}\right\} \subset \mathcal{F}, \exists w, z \in F \text { with } \operatorname{deg}(w)=1, \operatorname{deg}(z)=3\right\} \mid, \\
& m_{2}:=\mid\left\{F \in \mathcal{F}:\left\{x_{1}, x_{2}\right\} \subset \mathcal{F}, \exists w, z \in F \text { with } \operatorname{deg}(w)=\operatorname{deg}(z)=2\right\} \mid, \\
& m_{3}:=\mid\left\{F \in \mathcal{F}:\left\{x_{1}, x_{2}\right\} \subset \mathcal{F}, \exists w \in F \text { with } \operatorname{deg}(w)=4\right\} \mid,
\end{aligned}
$$

and observe that $m_{1}+m_{2}+m_{3}=s=14$. Note that $n_{20}=2,3 n_{3}=n_{1}=m_{1}, n_{2}=m_{2}$, and $4 n_{4}=3 m_{3}$. In particular, $m_{3}$ is even so $n_{1}+n_{2}=m_{1}+m_{2}$ is also even. Observe that

$$
\begin{aligned}
234 & =9 \cdot 26=k m=\sum_{x \in X} \operatorname{deg}(x)=20 n_{20}+8 n_{8}+4 n_{4}+3 n_{3}+2 n_{2}+n_{1} \\
& =20 n_{20}+8 n_{8}+4 n_{4}+n_{1}+2 n_{2}+n_{1}=20 n_{20}+8 n_{8}+4 n_{4}+2\left(n_{1}+n_{2}\right) .
\end{aligned}
$$

Since $n_{1}+n_{2}$ is even, (4.25) implies that $4 \mid 234$, which is a contradiction. 
$\epsilon \in\{2,3\}$ : Without loss of generality, we will assume $\operatorname{deg}\left(x_{1}\right) \leq \operatorname{deg}\left(x_{2}\right)$ or equivalently $\overline{\text { that } u \geq t}$. Observe that (3.16) and (3.17) imply

$$
\begin{aligned}
\operatorname{deg}\left(x_{1}\right) & =\sum_{x \in F_{1}} \operatorname{deg}(x)-\sum_{z \in F_{1} \backslash\left\{x_{1}\right\}} \operatorname{deg}(z) \geq \sum_{x \in F_{1}} \operatorname{deg}(x)-(k-1)^{2} \\
& =k^{2}+3 \epsilon-(k-1)^{2}=2 k-1+3 \epsilon .
\end{aligned}
$$

If $\epsilon \in\{2,3\}$, then $s \leq 16$ implies $u=6$. When $\epsilon=2$, we have $\operatorname{deg}\left(x_{1}\right)=21$, which contradicts (4.26). When $\epsilon=3$, we have $\operatorname{deg}\left(x_{1}\right)=22$, which again contradicts (4.26).

The case $k \in\{6,7,8\}$ can be eliminated with an argument similar to the one for $k=9, \epsilon \in\{2,3\}$; we omit the details.

Case Analysis for Case 2:

If $k=9$, then we arrive at a contradiction by using the third to last expression in (3.23).

If $k=8$, then $m \in\{20,21,22,23,24\}$ by $(3.23)$. For $m \in\{21,22,23,24,25\}$, any heavy vertex $x$ satisfies $\operatorname{deg}(x) \geq m-5$ by (3.10). Hence, the upper bound in (3.22) is improved and implies that $\operatorname{deg}\left(x_{1}\right)=s+t+w+x \leq 16$. This gives a contradiction for $m \in\{22,23,24\}$. For $m=21$, we have $s=6, w=x=y=5$, and $t=u=v=z=0$. Hence $\operatorname{codeg}\left(\left\{x_{i}, x_{j}\right\}\right)=11$ for $\{i, j\} \in\{1,2,3\}$. Adding the constraint $z_{11} \geq 3$ to (2.3) yields that $\left|\partial^{2} \mathcal{F}\right| \geq\left(\begin{array}{c}m \\ 2\end{array}\right)$, which contradicts our initial assumption; we omit the details since the computation is similar to that in (4.24).

If $k=7$, then $m \in\{16,17,18,19,20\}$ by (3.23) and (3.10) shows that any heavy vertex $x$ satisfies $\operatorname{deg}(x) \geq m-4$. Hence, the upper bound in (3.22) is improved and implies that $\operatorname{deg}\left(x_{1}\right)=s+t+w+x \leq 13$. This gives a contradiction for $m \in\{18,19,20\}$.

If $k=7$ and $m=17$, then we conclude $s=5, w=x=y=4$, and $t=u=v=z=0$. Note that if $x \in X$ is heavy then $\operatorname{deg}(x) \geq 13$ by (3.16). If there is a fourth heavy vertex $x_{4}$, it can be in at most one of the five sets on $x_{1}, x_{2}, x_{3}$; moreover since heavy vertices have degree at least thirteen, $x_{4}$ is in each of the four sets on $\left\{x_{1}, x_{2}\right\},\left\{x_{1}, x_{3}\right\}$, and $\left\{x_{2}, x_{3}\right\}$. As $\lambda=3$, this argument shows that there are at most four heavy vertices. If there are exactly four heavy vertices $x_{1}, x_{2}, x_{3}, x_{4}$, then codeg $\left(\left\{x_{i}, x_{j}\right\}\right)=9$ for $\{i, j\} \subset\{1,2,3,4\}$. Adding the constraint $z_{9} \geq 6$ to $(2.3)$ yields that $\left|\partial^{2} \mathcal{F}\right| \geq\left(\begin{array}{c}m \\ 2\end{array}\right)$, which contradicts our initial assumption; we omit the details since the computation is similar to the one in (4.24). Hence, there are exactly three heavy vertices and $\operatorname{deg}\left(x_{1}\right)=\operatorname{deg}\left(x_{2}\right)=\operatorname{deg}\left(x_{3}\right)=13$. Moreover, any set in $\mathcal{F}$ contains either exactly two or exactly three heavy vertices. Let $F^{\prime}$ be a set that contains exactly two heavy vertices. Equations (3.16) and (3.17) yield that $F^{\prime}$ contains four vertices of degree six and one of degree five. Hence, if $w$ is a light vertex and $w$ is contained in a set of $\mathcal{F}$ with two heavy vertices, then $\operatorname{deg}(w) \in\{5,6\}$; otherwise $\operatorname{deg}(w)=\operatorname{codeg}\left(x_{1}, x_{2}, x_{3}, w\right)=1$. Now let $\hat{F}$ be a set in $\mathcal{F}$ that contains exactly three heavy vertices. Since $\operatorname{deg}(w) \in\{1,5,6\}$ for $w \in X \backslash\left\{x_{1}, x_{2}, x_{3}\right\}$, (3.17) yields that $\hat{F}$ contains three vertices of degree five and one vertex of degree one. As $s=5$, there are fifteen vertices of degree five and five of degree one. Let $n_{i}$ denote the number of vertices 
of degree $i$. We have

$$
119=k m=\sum_{x \in X} \operatorname{deg}(x)=13 n_{13}+6 n_{6}+5 n_{5}+n_{1}=39+6 n_{6}+75+5,
$$

which implies that $n_{6}=0$, a contradiction.

If $k=7$ and $m=16$ then (3.23) implies that $s \in\{4,5\}$ and $t+u+v+2 z \leq 1$. We conclude that $z=0$ and at most one of $t, u, v$ equals one. We first show that the situation where exactly one of $t, u, v$ is one is impossible. Without loss of generality, assume for a contradiction that $t=1$ and $u=v=0$. If $s=4$, then (3.22) implies that

$$
16=m=s+t+u+v+w+x+y+z \leq 4+1+0+0+0+3+3+4=15,
$$

which is a contradiction. If $s=5$, then we can conclude via a similar argument that $w=x=3$ and $y=4$. Consider the unique $\hat{F} \in \mathcal{F}$ with $x_{1} \in F$ and $x_{2}, x_{3} \notin F$. Since light vertices have degree at most six, $\hat{F}$ must contain another heavy vertex $x_{4}$ by (3.17). Now $\operatorname{deg}\left(x_{4}\right) \geq 12$ and since $x_{4}$ can only be in one of the five sets on $x_{1}, x_{2}, x_{3}$, we have that $x_{4}$ is in each of the remaining 11 sets. As a result, for $i, j \in\{1,2,3,4\}$, we have codeg $\left(\left\{x_{i}, x_{j}\right\}\right)=8$ if $\{i, j\} \neq\{1,3\}$ and we have codeg $\left(\left\{x_{1}, x_{3}\right\}\right)=9$. Adding the constraints $z_{9} \geq 1$ and $z_{8} \geq 5$ to $(2.3)$ yields that $\left|\partial^{2} \mathcal{F}\right| \geq\left(\begin{array}{c}m \\ 2\end{array}\right)$, which contradicts our initial assumption; we omit the details since the computation is similar to the one in (4.24). Hence, we can assume $t=u=v=0$. If $s=5$, then two of $w, x, y$ equal four and the other equals three. Hence, two of the pairs $\left\{x_{1}, x_{2}\right\},\left\{x_{1}, x_{3}\right\} .\left\{x_{2}, x_{3}\right\}$ have codegree nine and one has codegree eight. Adding the constraints $z_{9} \geq 2$ and $z_{8} \geq 1$ to (2.3) yields that $\left|\partial^{2} \mathcal{F}\right| \geq\left(\begin{array}{c}m \\ 2\end{array}\right)$, which contradicts our initial assumption; we omit the details since the computation is similar to the one in (4.24). If $s=4$, then $w=x=y=4$ so $\operatorname{deg}\left(x_{1}\right)=\operatorname{deg}\left(x_{2}\right)=\operatorname{deg}\left(x_{3}\right)=12$ and $\operatorname{codeg}\left(\left\{x_{i}, x_{j}\right\}\right)=8$ for $\{i, j\} \subset\{1,2,3\}$. If we add the constraint $z_{8} \geq 3$ to $(2.3)$, then the corresponding dual linear program is

$$
\begin{array}{r}
\text { Maximize } \begin{array}{r}
360 w+336 x+y \\
\text { subject to: } \quad\left(\begin{array}{l}
i \\
2
\end{array}\right) w+i x \leq 1, \quad i \in[16] \backslash\{8\} \\
\left(\begin{array}{l}
8 \\
2
\end{array}\right) w+8 x+y \leq 1 . \\
y \geq 0 .
\end{array}
\end{array}
$$

It is not difficult to see that $(-1 / 3,2 / 3,5)$ is a feasible solution to $(4.27)$ so $\left|\partial^{2} \mathcal{F}\right| \geq 119$. Since $\left(\begin{array}{c}16 \\ 2\end{array}\right)=120$, we obtain a contradiction unless $(-1 / 3,2 / 3,5)$ is an optimal solution to (4.27). If $(-1 / 3,2 / 3,5)$ is an optimal solution to $(4.27)$, then by complementary slackness $\operatorname{codeg}(\{x, y\}) \in\{0,2,3,8\}$ for $\{x, y\} \in\left(\begin{array}{c}X \\ 2\end{array}\right)$; moreover $z_{8}=3$. Since $t=u=v=z=0$, if $w \in X \backslash\left\{x_{1}, x_{2}, x_{3}\right\}$ then

$$
\operatorname{deg}(w)=\sum_{\{i, j\} \subset\{1,2,3\}} \operatorname{codeg}\left(\left\{x_{i}, x_{j}, w\right\}\right)-2 \operatorname{codeg}\left(\left\{x_{1}, x_{2}, x_{3}, w\right\}\right) \leq 9,
$$


so (3.16) shows that $x_{1}, x_{2}, x_{3}$ are the only heavy vertices. Moreover, as $t=u=v=z=0$, every $F \in \mathcal{F}$ contains either exactly two or exactly three heavy vertices. If $F^{\prime} \in \mathcal{F}$ is a set with exactly two heavy vertices, then (3.16) and (3.17) yield that either $F^{\prime}$ contains three vertices of degree six and two vertices of degree five or four vertices of degree six and one of degree four. Now, every light vertex $w$ must be contained in a set with exactly two heavy vertices; otherwise $\operatorname{deg}(w)=\operatorname{codeg}\left(x_{1}, x_{2}, x_{3}, w\right)=1$, which contradicts the fact that codeg $(\{x, y\}) \in\{0,2,3,8\}$. Hence, $\operatorname{deg}(w) \in\{4,5,6\}$ for $w \in X \backslash\left\{x_{1}, x_{2}, x_{3}\right\}$. As a result, if $\hat{F}$ is a set with three heavy vertices, then (3.17) yields that $\hat{F}$ contains four vertices of degree four. We conclude that $\operatorname{deg}(w) \in\{4,6\}$ for $w \in X \backslash\left\{x_{1}, x_{2}, x_{3}\right\}$. Since $s=4$, there are sixteen vertices of degree four. Let $n_{i}$ denote the number of vertices of degree $i$; we have

$$
112=k m=\sum_{x \in X} \operatorname{deg}(x)=12 n_{12}+6 n_{6}+4 n_{4}=36+6 n_{6}+64
$$

so $n_{6}=2$, which is impossible.

If $k=6$, then $\epsilon \in\{1,2\}$ by Theorem 3.1. Let $n_{i}$ denote the number of vertices of degree $i$. If $\epsilon=2$, then $n_{1}=2, n_{4}=9$, and $n_{10}=4$ by a result of Vanstone [15]. Using (3.17), we see that $\mathcal{F}$ is uniquely determined and must be the family

$$
\begin{aligned}
& \{\{1,2,3,5,6,7\},\{1,2,3,8,9,10\},\{1,2,3,11,12,13\},\{1,2,4,5,8,11\},\{1,2,4,6,9,12\}, \\
& \{1,2,4,7,10,13\},\{1,3,4,5,10,12\},\{1,3,4,6,8,13\},\{1,3,4,7,9,11\},\{2,3,4,5,9,13\}, \\
& \{2,3,4,6,10,11\},\{2,3,4,7,8,12\},\{1,2,3,4,14,15\}\} .
\end{aligned}
$$

Hence, $\left|\partial^{2} \mathcal{F}\right|=87>\left(\begin{array}{c}13 \\ 2\end{array}\right)$. If $k=6$ and $\epsilon=1$, then either $n_{1}=2, n_{3}=3, n_{4}=6, n_{9}=3$, and $n_{10}=1$ or $n_{4}=9$ and $n_{9}=4$ by a result of Vanstone [15]. Again using (3.17), we can conclude that $\mathcal{F}$ is uniquely determined in both cases. In the first case, $\mathcal{F}$ must be

$$
\begin{aligned}
& \{\{1,2,4,5,6,11\},\{1,2,4,7,8,12\},\{1,2,4,9,10,13\},\{1,3,4,7,9,11\}, \\
& \{1,3,4,5,10,12\},\{1,3,4,6,8,13\},\{2,3,4,8,10,11\},\{2,3,4,6,9,12\} \text {, } \\
& \{2,3,4,5,7,13\},\{1,2,3,5,8,9\},\{1,2,3,6,7,10\},\{1,2,3,4,14,15\}\} \text {; }
\end{aligned}
$$

hence, $\left|\partial^{2} \mathcal{F}\right|=84>\left(\begin{array}{c}12 \\ 2\end{array}\right)$. In the latter case, $\mathcal{F}$ is the complement of a projective plane of order 3 with respect to a line; hence $\left|\partial^{2} \mathcal{F}\right|=78>\left(\begin{array}{c}12 \\ 2\end{array}\right)$.

ACKnowledgement: The work in this paper benefited from a number of discussions with Eva Czabarka, Ida Kantor, Gyula O.H. Katona, Nathan Lemons, Balázs Patkós, László Székely, and Jacques Verstraëte. The author thanks the NSF for funding her and IPAM for hosting her while she was a core participant in the CMA 2009 program. The author also thanks the US State Department and the Hungarian Fulbright Commission for funding her and the Rényi Institute for hosting her while she was a Fulbright fellow. Finally, the author thanks the anonymous referee for carefully reading this manuscript. 


\section{References}

[1] L. Babai. On the nonuniform Fisher inequality. Discrete Math., 66(3):303-307, 1987.

[2] L. Babai and P. Frankl. Linear algebra methods in combinatorics. manuscript, 1992.

[3] W. S. Connor, Jr. On the structure of balanced incomplete block designs. Ann. Math. Statistics, 23:57-71, 1952.

[4] N. G. de Bruijn and P. Erdős. On a combinatorial problem. Nederl. Akad. Wetensch., Proc., 51:1277-1279 = Indagationes Math. 10, 421-423 (1948), 1948.

[5] M. Deza. Une propriété extrémale des plans projectifs finis dans une classe de codes équidistants. Discrete Math., 6:343-352, 1973.

[6] M. Deza. Solution d'un problème de Erdős-Lovász. J. Combinatorial Theory Ser. B, 16:166-167, 1974.

[7] P. Frankl and Z. Füredi. A sharpening of Fisher's inequality. Discrete Math., 90(1):103-107, 1991.

[8] B. Gardner. On coverings and $(r, \lambda)$-systems. PhD thesis, University of Waterloo, 1972.

[9] B. Gardner and S. A. Vanstone. Some results on irreducible $(r, \lambda)$-designs. Utilitas Math., 18:291-300, 1980.

[10] J. I. Hall. On two conjectures of Stanton and Mullin. J. Combinatorial Theory Ser. A, 22(2):153-162, 1977.

[11] K. Majindar. On the parameters and intersection of blocks of balanced incomplete block designs. Ann. Math. Statist., 33:1200-1205, 1962.

[12] D. McCarthy and S. A. Vanstone. On the structure of regular pairwise balanced designs. Discrete Math., 25(3):237-244, 1979.

[13] H. J. Ryser. An extension of a theorem of de Bruijn and Erdös on combinatorial designs. J. Algebra, 10:246-261, 1968.

[14] R. G. Stanton and R. C. Mullin. Inductive methods for balanced incomplete block designs. Ann. Math. Statist., 37:1348-1354, 1966.

[15] S. A. Vanstone. Irreducible regular pairwise balanced designs. Utilitas Math., 15:249259, 1979.

[16] D. R. Woodall. Square $\lambda$-linked designs: A survey. In Combinatorial Mathematics and its Applications (Proc. Conf., Oxford, 1969), pages 349-355. Academic Press, London, 1971. 